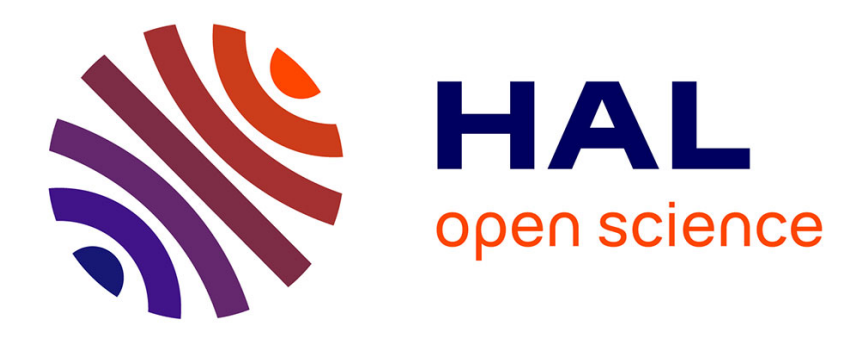

\title{
Abdominal arteries recognition in X-Ray using a structural model
}

Olivier Nempont, Raoul Florent

\section{To cite this version:}

Olivier Nempont, Raoul Florent. Abdominal arteries recognition in X-Ray using a structural model. SPIE Medical Imaging 2010: Image Processing, Feb 2010, San Diego, CA, United States. pp.76230G, 10.1117/12.844331 . hal-00508890

\section{HAL Id: hal-00508890 https://hal.science/hal-00508890}

Submitted on 6 Aug 2010

HAL is a multi-disciplinary open access archive for the deposit and dissemination of scientific research documents, whether they are published or not. The documents may come from teaching and research institutions in France or abroad, or from public or private research centers.
L'archive ouverte pluridisciplinaire HAL, est destinée au dépôt et à la diffusion de documents scientifiques de niveau recherche, publiés ou non, émanant des établissements d'enseignement et de recherche français ou étrangers, des laboratoires publics ou privés. 


\title{
Abdominal arteries recognition in X-Ray using a structural model
}

\author{
Olivier Nempont, Raoul Florent \\ Medisys Research Lab, Philips Healthcare, 33 rue de Verdun, 92156 Suresnes, France
}

\begin{abstract}
The automatic recognition of vascular trees is a challenging task, required for roadmapping or advanced visualization. For instance, during an endovascular aneurysm repair (EVAR), the recognition of abdominal arteries in angiograms can be used to select the appropriate stent graft. This choice is based on a reduced set of arteries (aorta, renal arteries, iliac arteries) whose relative positions are quite stable.

We propose in this article a recognition process based on a structural model. The centerlines of the target vessels are represented by a set of control points whose relative positions are constrained. To find their position in an angiogram, we enhance the target vessels and extract a set of possible positions for each control point. Then, a constraint propagation algorithm based on the model prunes those sets of candidates, removing inconsistent ones. We present preliminary results on 5 cases, illustrating the potential of this approach and especially its ability to handle the high variability of the target vessels.
\end{abstract}

Keywords: Medical image interpretation, automatic recognition, structural model, abdominal arteries, X-Ray imaging.

\section{INTRODUCTION}

Endovascular aneurysm repair (EVAR) is a minimally invasive surgical intervention for the treatment of abdominal aortic aneurysms (AAA), i.e. a widening of the aorta below the renal arteries. The interventionist introduces a stent graft through the femoral arteries under X-Ray guidance. This endoprothesis is then deployed just below the renal arteries and above the common iliac bifurcation. Therefore the choice of the appropriate stent graft depends on the subject anatomy and is based on a reduced set of arteries (aorta, renal arteries, iliac arteries). The intervention planning could thus benefit from the automatic interpretation of abdominal angiography. For instance approaches were proposed to automatically or semi-automatically segment the abdominal aorta in CTA or MRA, e.g. in ${ }^{1-3}$. The diameter of the lumen or of the outer wall are then estimated along the aorta, providing

Further author information: (Send correspondence to O. Nempont)

O. Nempont: E-mail: olivier.nempont@philips.com, Telephone: +33 (0)1 47283657

R. Florent: E-mail: raoul.florent@philips.com, Telephone: +33 (0)1 47283548
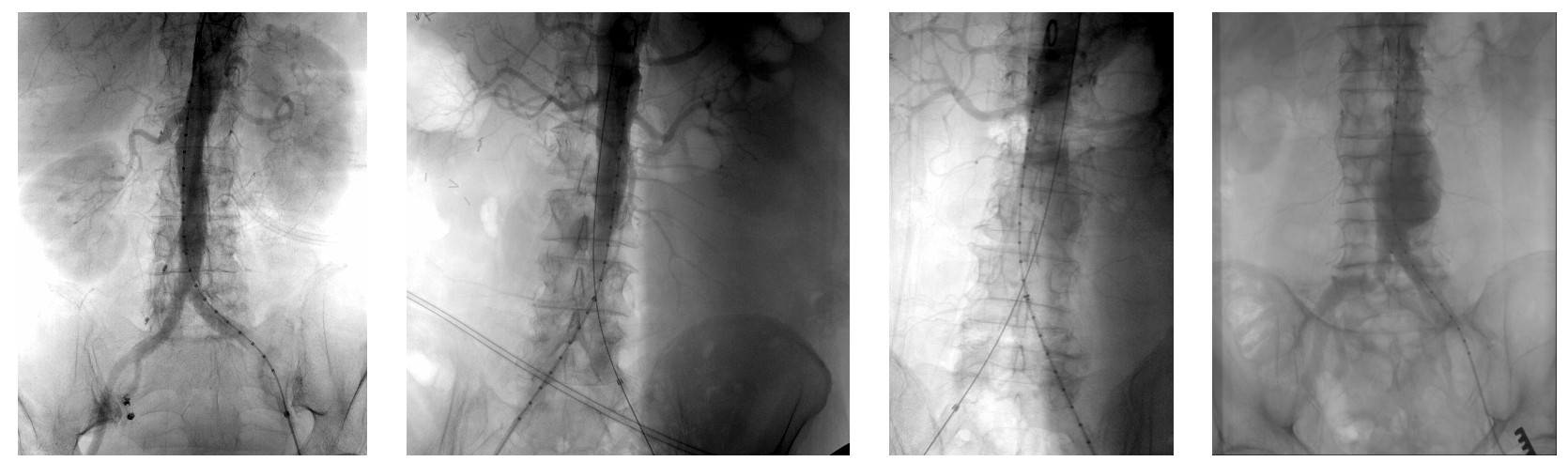

Figure 1. Angiograms of abdominal aorta. 


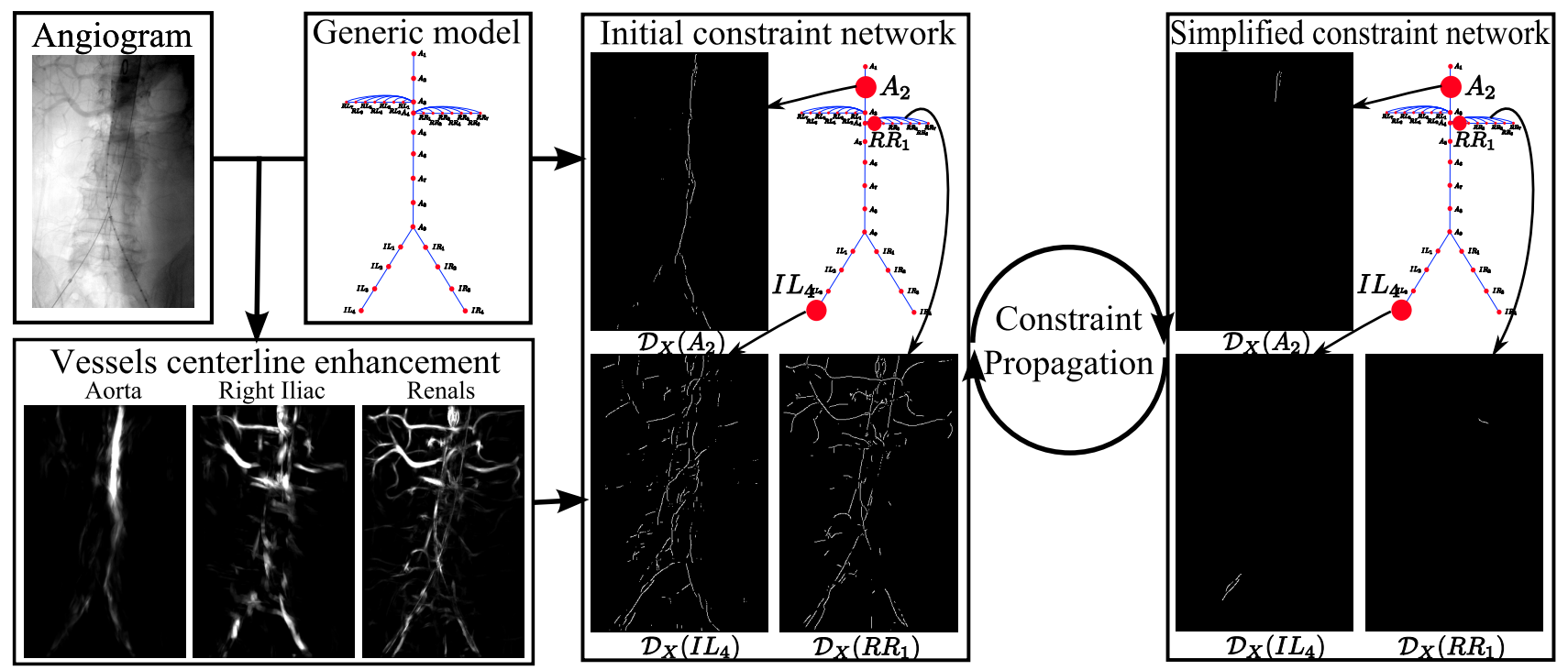

Figure 2. Synthetic view of the proposed approach.

relevant information to assess the risk of rupture. However to the best of our knowledge, automatic interpretation processes including the collateral arteries were not proposed.

During the intervention, several angiograms are performed in order to validate the choice of the stent graft and then to position and to deploy the device. Four angiograms of different subjects are shown in figure 1 . Their aneurysms are located on the aorta (the large vertical dark vessel) between the renal arteries (the lateral vessels in the upper part of the angiogram) and the iliac arteries (the two branches in the lower part). We are interested here in the automatic recognition of the main arteries in those angiograms.

To automatically perform the recognition, a model of the target anatomy has to be used. Due to the high variability of shapes, the registration of an iconic atlas ${ }^{4}$ may not be robust. Statistical shape model ${ }^{5,6}$ could deal with this variability but the available optimization schemes generally require a good initialization of the pose parameters. On the other hand, approaches based on structural models ${ }^{7}$ and constraint propagation ${ }^{8}$ were proposed in ${ }^{9,10}$ to perform the recognition of brain structures. Those models represent the structures to be recognized, some of their properties and their relations (in particular their relative position). To obtain a solution (i.e. to assign regions of the image to the structures of the model), the constraints propagation algorithms proposed in ${ }^{9-11}$ iteratively prune the set of possible regions for each structure, removing the ones that are inconsistent with the structural model. We propose here to use a structural model to perform the recognition of abdominal arteries. It represents the vessel centerlines by a set of control points and their relative positions. We then propose a constraint propagation algorithm to obtain the position of the model control points in an angiogram.

The proposed approach is illustrated in Figure 2. First the vessel centerlines are enhanced using ridge filters. Then the matching of the anatomical model is performed by a constraint propagation algorithm. In Section 2 , the structural model representing the target abdominal arteries is presented. In Section 3, we briefly describe the filtering process used to enhance the vessel centerlines and to extract possible positions for the model control points. Section 4 is the core of this paper. It presents the constraint network derived from the model. The chosen mathematical formulation of the constraints is then detailed along with operators that prune inconsistent candidates according to the constraints. The operators are iteratively applied to achieve constraint propagation. Finally in Section 5, we present some preliminary results.

\section{STRUCTURAL MODEL}

To perform the recognition task, we rely on the structural model of the abdominal arteries illustrated in Figure 3. The centerlines of the target abdominal arteries are represented by a set of control points. For in- 
stance, the aorta is represented by 9 control points $\left(A_{1} \ldots A_{9}\right)$ in that case. $A_{9}$ represents the aortic bifurcation. $A_{3}$ and $A_{4}$ are respectively the points of the aorta in front of the branching points of the left and right renal arteries $\left(R L_{1}\right.$ and $\left.R R_{1}\right)$. In fact the control points are associated with specific anatomical points and their relative position is quite stable. For instance $A_{i}$ is below $A_{i-1} . R L_{1}$ is on the left of $A_{3}$ and their distance is almost equal to the aorta radius.

This knowledge is represented in the model by a set of relations between the control points on their relative position. We consider 3 types of relations:

- Directional relations between two control points represent a prior on the orientation of their relative position. Those relations are characterized by a direction and a tolerance around that direction that are defined in the coordinate system of the X-Ray imaging system or with respect to the local vessel orientation.

- Distance relations between two control points represent a prior on their distance. It can be expressed as an interval in centimeters or with respect to the local vessel diameter.

- Geodesic distance relations are defined between control points that belong to the same vessel. They assume that the geodesic distance with respect to the vessel has to be smaller than a given value (related to the upper endpoint of the distance interval in a distance constraint).

In addition we include priors on the radius and on the orientation of the vessels. For instance abdominal aorta's diameter normally ranges from $1.4 \mathrm{~cm}$ to $2.4 \mathrm{~cm}$ and is quite vertical in the X-Ray images. As the subrenal aorta normally contains an aneurysm, we consider a wider interval on this part. Thus for each control points, we include a prior on the local diameter and orientation of the vessel.

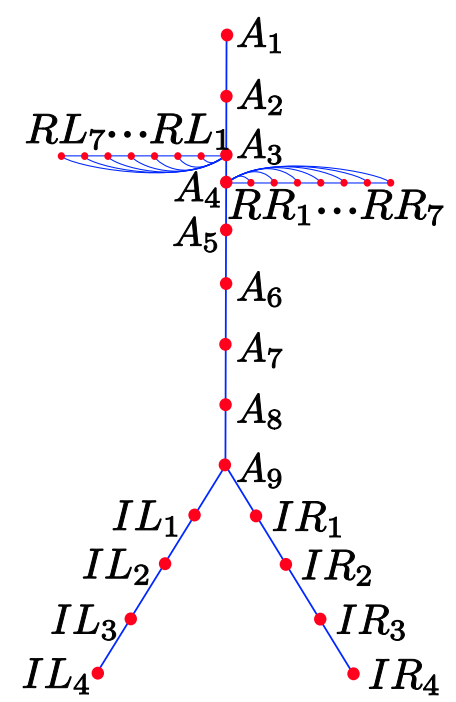

Figure 3. Structural model of the abdominal arteries.

\section{CANDIDATES EXTRACTION}

For each control point, we want to extract a set of possible positions. As control points represent centerline points of dark vessels (whose radius and orientation are roughly known), we first apply multi-scale ridge filters ${ }^{12-14}$ to enhance the centerlines of ridges whose radius and orientation fit the prior hold by the model. Besides a ridgeness score, we also obtain the optimal local orientation and radius. From those maps, we then extract a set of possible positions for each control point.

This process is illustrated in figure 4. In (d) and (e) we consider the control points representing the renal arteries. The ridgeness map is presented in (d). The associated set of possible positions is shown in (e). Those candidates are then associated with all the control points representing the renal arteries.

\section{CONSTRAINT PROPAGATION}

\subsection{General definitions on constraint propagation}

We recall here the definitions and notations used in the sequel. Comprehensive surveys on constraint networks and constraint propagation can be found for instance in ${ }^{8,15,16}$. A constraint network is defined by a triplet $N=\langle\chi, \mathcal{D}, \mathcal{C}\rangle$ where:

- $\chi=\left\{x_{1}, \ldots, x_{n}\right\}$ is the set of variables of our problem,

- $\mathcal{D}$ is the set of domains associated with those variables. A variable $x_{i} \in \chi$ takes values in the domain $\mathcal{D}\left(x_{i}\right)$, - $\mathcal{C}$ is the set of constraints. Each constraint $C=(\operatorname{vars}(C), \operatorname{rel}(C))$ involves a set of variables $\operatorname{var} s(C) \subseteq \chi$ and is represented by a relation $\operatorname{rel}(C)$ defined on the Cartesian product of their domains.

An instantiation $I=\left\{\left(x_{1}, v_{1}\right), \ldots,\left(x_{k}, v_{k}\right)\right\}$ on the variables $Y=\left\{x_{1}, \ldots, x_{k}\right\} \subseteq \chi$ is valid if $\forall x_{i} \in Y, v_{i} \in$ $\mathcal{D}\left(x_{i}\right)$. I satisfies a constraint $C$ such that $\operatorname{var} s(C) \subseteq Y$ if $I[\operatorname{vars}(C)] \in \operatorname{rel}(C)$, where $I[\operatorname{vars}(C)]$ is the projection of $I$ on $\operatorname{var} s(C)$. $I$ is locally consistent if $I$ is valid and if for each constraint $C \in \mathcal{C}$ such that $\operatorname{var} s(C) \subseteq Y, I$ satisfies $C$. A solution of the constraint network $N$ is a locally consistent instantiation $I$ on $\chi$. We denote by $\operatorname{sol}(N)$ the set of solutions of $N$. A constraint network is said satisfiable if it has at least one solution.

Efficient backtracking algorithms ${ }^{17,18}$ were proposed to obtain solutions of a constraint network. However many problems cannot be solved by those algorithms for complexity reasons. To simplify the problem a constraint 


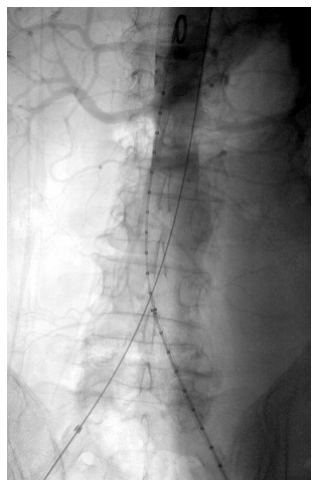

(a)

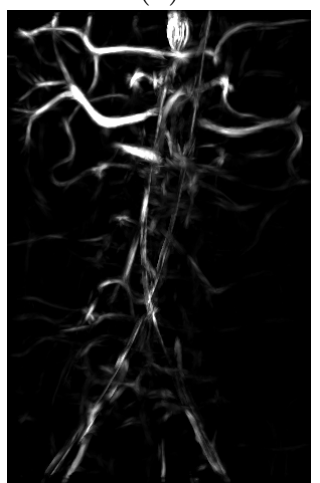

(d)

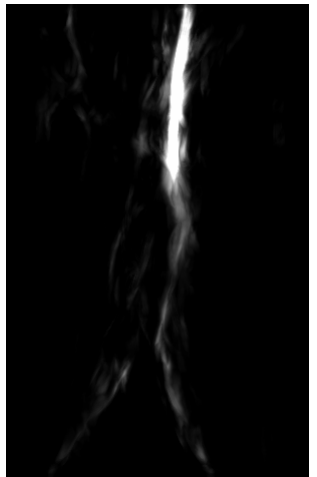

(b)

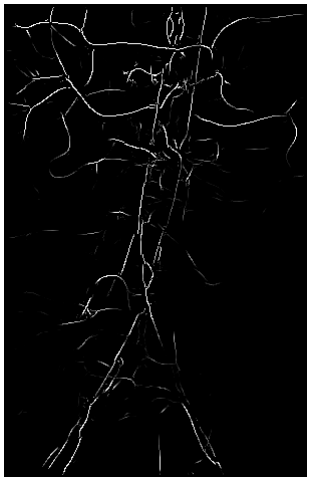

(e)

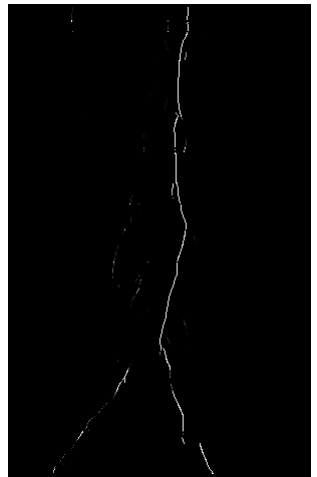

(c)

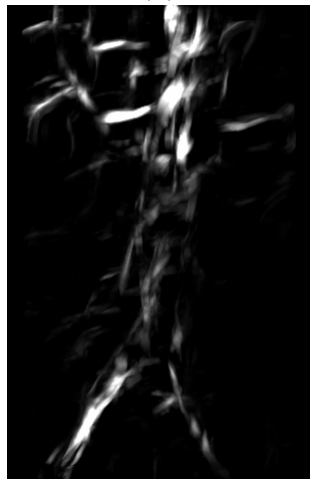

(f)

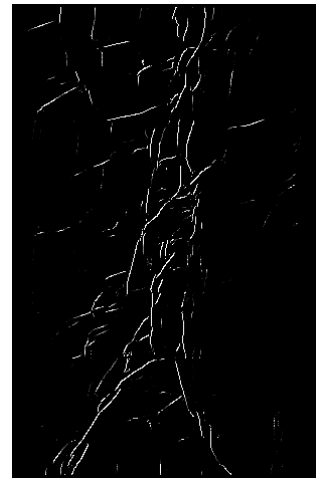

$(\mathrm{g})$

Figure 4. (a) Angiogram. Ridgeness map considering control points on the aorta (b), on the renal arteries (d) and on the left iliac artery (f). (c-e-g) Associated sets of candidates.

propagation algorithm ${ }^{8}$ can first be applied. It iteratively transforms an initial constraint network $N$ into a simpler network $N^{\prime}$ that present the same solutions by: (i) reducing the domains of the variables, (ii) inferring new constraints. We give below the main definitions and notations related to domain based constraint propagation. Let $N=\langle\chi, \mathcal{D}, \mathcal{C}\rangle$. The set $\mathcal{P}_{N D}$ of all domain-based tightenings (or reductions) of $N$ is the set of networks $\left\{N^{\prime}=\left\langle\chi, \mathcal{D}^{\prime}, \mathcal{C}\right\rangle\right\}$ such that $\mathcal{D}^{\prime} \subseteq \mathcal{D}$. We denote by $\leq_{N}$ the partial ordering associated with the domain inclusion relation. The set $\mathcal{P}_{N D}^{s o l}$ is the subset of $\mathcal{P}_{N D}$ of networks that present the same solutions as $N$, i.e. $\forall N^{\prime} \in \mathcal{P}_{N D}^{s o l}, \operatorname{sol}\left(N^{\prime}\right)=\operatorname{sol}(N)$.

$\mathcal{P}_{N D}^{s o l}$ has a least element denoted by $G_{N D}$ whose domains contain only values belonging to a solution. As computing $G_{N D}$ is NP-hard, domain-based constraint propagation aims at getting an element of $\mathcal{P}_{N D}^{\text {sol }}$, as small as possible in polynomial time. Such a process iteratively removes values that cannot belong to a solution, for instance by applying propagators. A propagator $f$ is an operator associated with a constraint $C \in \mathcal{C}$. It tightens the domains $\left(\forall N^{\prime} \in \mathcal{P}_{N D}, f\left(N^{\prime}\right) \in \mathcal{P}_{N^{\prime} D}\right)$ regardless of the other constraints. $f$ is:

- correct if $\forall N^{\prime} \in \mathcal{P}_{N D}^{\text {sol }}, f\left(N^{\prime}\right) \in \mathcal{P}_{N^{\prime} D}^{\text {sol }}$,

- increasing if $\forall N_{1}, N_{2} \in \mathcal{P}_{N D}, N_{1} \leq_{N} N_{2} \Rightarrow f\left(N_{1}\right) \leq_{N} f\left(N_{2}\right)$,

- idempotent if $\forall N^{\prime} \in \mathcal{P}_{N D}, f\left(f\left(N^{\prime}\right)\right)=f\left(N^{\prime}\right)$.

A constraint propagation process that iteratively applies a set of propagators ends up when no propagator can further reduce a domain. If the propagators are increasing (this is generally the case), the result does not depend on the order of application of the propagators, and is then called the least fixed point.

In the following a propagator $f$ will then be described as follows:

$$
\frac{\langle\operatorname{vars}(C) ; \mathcal{D} ; C\rangle}{\left\langle\operatorname{vars}(C) ; \mathcal{D}^{\prime} ; C\right\rangle}
$$

where $\mathcal{D}$ and $\mathcal{D}^{\prime}$ are the domains associated with the variables $\operatorname{vars}(C)$ and $\mathcal{D}^{\prime} \leq_{N} \mathcal{D}$. 


\subsection{Constraint network based on the anatomical model}

Based on local characteristics, we have associated a set of possible positions with each control point. To prune those candidates, we rely on the spatial relations hold by the model. To this aim, we derive a constraint network ${ }^{16}$ from the structural model and we set up a constraint propagation algorithm to remove as many inconsistent candidates as possible according to the spatial relations of the model.

We denote by $N=\langle\chi, \mathcal{D}, \mathcal{C}\rangle$ the constraint network. Each control point of the model is associated with a variable $n \in \chi$. As illustrated besides, a variable $n$ represents the position of the control point, the local orientation and the radius of the vessel to which it belongs. A value for $n$ is then the combination of a position $x$ in the image spatial domain $X \subseteq \mathbb{Z}^{2}$, a local orientation $\vec{d}$ and a radius $s$. Therefore each variable takes values in a

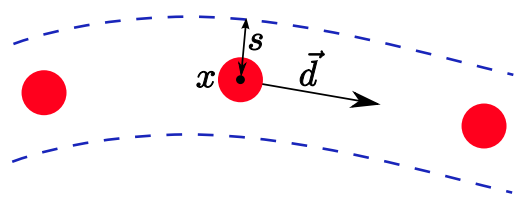
domain $\mathcal{D}(n)$ which is a subset of $\mathbb{Z}^{2} \times\left[-\pi, \pi\left[\times \mathbb{R}^{+}\right.\right.$.

We limit the set of possible values of $n$ to the set of candidates previously obtained (cf Section 3 ). This defines the initial domain of $n$. As the low-level filters also provide the optimal angle $\vec{d} \in[0, \pi[$ and radius $s$, the initial domain contains for each possible position $x$ the values $(x, \vec{d}, s)$ and $(x,-\vec{d}, s)$. Therefore the domains can be represented on $X \times\{0,1\}$. The constraint propagation algorithm will iteratively tighten the domains. If during the process a domain is empty, we can conclude that the problem is not satisfiable.

The constraints $\mathcal{C}$ of $N$ are obtained from the relations of the model. For instance if the model contains a distance relation between $n_{1}$ and $n_{2}$, we define a constraint $C$ satisfied by values $\left(x_{1}, \overrightarrow{d_{1}}, s_{1}\right)$ and $\left(x_{2}, \overrightarrow{d_{2}}, s_{2}\right)$ such that $\left\|x_{2}-x_{1}\right\|$ belongs to a given interval. A value of $n_{1}$ (resp. $n_{2}$ ) that does not satisfy the constraint with any value in $\mathcal{D}\left(n_{2}\right)$ (resp. $\mathcal{D}\left(n_{1}\right)$ ), is inconsistent with respect to $C$ and can be remove from the domain. In fact that value cannot belong to a solution. With each constraint $C$ we associate a propagator that tightens the domains by removing inconsistent values. In Section 4.3, we present the chosen mathematical formulation of the constraints and the associated propagators.

\subsection{Constraints}

\subsubsection{Directional constraint}

The anatomical model contains relations to represent stable directional relative positions between control points as highlighted in Section 2. To model the associated constraints, we rely on the mathematical morphology approach* introduced in ${ }^{19}$, that characterize a directional relation by a direction $\overrightarrow{u_{d}}$ and an angle $k$ representing a tolerance around $\overrightarrow{u_{d}}$. With respect to the origin of space, a point $x$ is in the specified direction if $\arccos \frac{\mathbf{x} \cdot \overrightarrow{u_{d}}}{\|\mathbf{x}\|} \leq k$. If we denote by $\nu$ a structuring element associated with that condition, the dilation $\delta_{\nu}(y)$ represents the set of points that are in the specified direction with respect to a point $y$. Therefore we consider that two points $x_{1}$ and $x_{2}$ satisfy the directional relation if $x_{2} \in \delta_{\nu}\left(x_{1}\right)$.

Definition 4.1 (Directional Constraint). Let $A$ and $B$ be two control points of the model satisfying a stable directional relative position characterized by a structuring element $\nu$. The constraint $C_{A, B}^{\nu}$ is defined as follows:

$$
\begin{aligned}
& \operatorname{rel}\left(C_{A, B}^{\operatorname{dir} \nu}\right): \quad \mathcal{D}(A) \times \mathcal{D}(B) \quad \rightarrow\{0,1\} \\
& \left(\left(x_{1}, \theta_{1}, \sigma_{1}\right),\left(x_{2}, \theta_{2}, \sigma_{2}\right)\right) \mapsto \begin{cases}1 & \text { if } x_{2} \in \delta_{\nu}\left(x_{1}\right), \\
0 & \text { otherwise. }\end{cases}
\end{aligned}
$$

An instantiation $I=\left\{\left(A,\left(x_{A}, \theta_{A}, \sigma_{A}\right)\right),\left(B,\left(x_{B}, \theta_{B}, \sigma_{B}\right)\right)\right\}$ that does not satisfy the condition $x_{B} \in \delta_{\nu}\left(x_{A}\right)$ is inconsistent with respect to $C_{A, B}^{\text {dir } \nu}$ and cannot be extended to a solution. Moreover a value $\left(x_{A}, \theta_{A}, \sigma_{A}\right)$ of $A$ that only belongs to inconsistent instantiations $\left(\forall(x, \theta, \sigma) \in \mathcal{D}(B),\left\{\left(A,\left(x_{A}, \theta_{A}, \sigma_{A}\right)\right),(B,(x, \theta, \sigma))\right\}\right.$ is inconsistent) cannot belong to a solution and can be removed from $\mathcal{D}(A)$. Therefore we associate with $C_{A, B}^{\text {dir }}{ }^{\nu}$ a propagator that removes as many inconsistent values as possible from $\mathcal{D}(A)$ and $\mathcal{D}(B)$.

\footnotetext{
${ }^{*} \mathrm{In}^{19}$ the authors propose a fuzzy representation. We consider here the binary version.
} 
A value $(x, \theta, \sigma)$ of $B$ exclusively belongs to inconsistent instantiations with respect to $C_{A, B}^{\operatorname{dir} \nu}$ if:

$$
\begin{aligned}
& \forall\left(x_{A}, \theta_{A}, \sigma_{A}\right) \in \mathcal{D}(A), x \notin \delta_{\nu}\left(x_{A}\right) \\
& \Leftrightarrow x \notin \cup_{x_{A} \in \mathcal{D}_{X}(A)} \delta_{\nu}\left(x_{A}\right) \\
& \Leftrightarrow x \notin \delta_{\nu}\left(\mathcal{D}_{X}(A)\right),
\end{aligned}
$$

where $\mathcal{D}_{X}(A)$ stands for the projection of $\mathcal{D}(A)$ on $X$. Therefore the propagator can remove from $\mathcal{D}(B)$ all values fulfilling that condition. Similarly the propagator can remove from $\mathcal{D}(A)$ all values $(x, \theta, \sigma)$ such that $x \notin \delta_{\breve{\nu}}\left(\mathcal{D}_{X}(B)\right)$, where $\breve{\nu}$ stands for the symmetric of $\nu$.

Definition 4.2 (Directional CONSTRAint PROPAGATOR). The propagator $f_{C_{A, B}^{d i r} \nu}$ associated with the directional relative position constraint between two control points $A$ and $B$ is defined as follows:

$$
\frac{\left\langle A, B ; \mathcal{D}(A), \mathcal{D}(B) ; C_{A, B}^{\operatorname{dir} \nu}\right\rangle}{\left\langle A, B ; \mathcal{D}(A) \cap \delta_{\breve{\nu}}\left(\mathcal{D}_{X}(B)\right) \times\left[-\pi, \pi\left[\times \mathbb{R}^{+}, \mathcal{D}(B) \cap \delta_{\nu}\left(\mathcal{D}_{X}(A)\right) \times\left[-\pi, \pi\left[\times \mathbb{R}^{+} ; C_{A, B}^{\text {dir } \nu}\right\rangle\right.\right.\right.\right.}
$$

\subsubsection{Distance constraint}

To model distance constraints, we also rely on a morphological formulation as it allows a simple expression of the associated propagator. If the model contains the relation "the distance between the control points $A$ and $B$ has to belong to $\left[d_{1}, d_{2}\right]$ ", we add the following constraint to the constraint network.

Definition 4.3 (Distance COnstraint). Let $A$ and $B$ be two control points whose distance belongs to the interval $\left[d_{1}, d_{2}\right]$. The constraint $C_{A, B}^{\text {dist }}\left[d_{1}, d_{2}\right]$ is defined as follows:

$$
\begin{array}{rlrl}
\operatorname{rel}\left(C_{A, B}^{\text {dist }\left[d_{1}, d_{2}\right]}\right): & \mathcal{D}(A) \times \mathcal{D}(B) & \rightarrow\{0,1\} \\
\left(\left(x_{1}, \theta_{1}, \sigma_{1}\right),\left(x_{2}, \theta_{2}, \sigma_{2}\right)\right) & \mapsto \begin{cases}1 & \text { if } x_{2} \in \delta_{\mathcal{B}_{d_{2}} \backslash \mathcal{B}_{d_{1}}}\left(x_{1}\right), \\
0 & \text { otherwise, }\end{cases}
\end{array}
$$

where $\mathcal{B}_{d}$ stands for the binary ball of radius $d$ and $\delta_{\mathcal{B}_{d}}$ is the dilation by $\mathcal{B}_{d}$.

As in the case of directional constraints, we obtain a propagator that removes inconsistent values from the domains of $A$ and $B$.

Definition 4.4 (Distance CONSTRAint PROPAGATOR). The propagator $f_{C_{A, B}^{\text {dist }\left[d_{1}, d_{2}\right]}}$ associated with the distance constraint between two control points $A$ and $B$ is defined as follows:

$$
\left.\frac{\left\langle A, B ; \mathcal{D}(A), \mathcal{D}(B) ; C_{A, B}^{\text {dist }\left[d_{1}, d_{2}\right]}\right\rangle}{\left\langle A, B ; \mathcal{D}(A) \cap \delta_{\mathcal{B}_{d_{2}} \backslash \mathcal{B}_{d_{1}}}\left(\mathcal{D}_{X}(B)\right) \times\left[-\pi, \pi\left[\times \mathbb{R}^{+}, \mathcal{D}(B) \cap \delta_{\mathcal{B}_{d_{2}} \backslash \mathcal{B}_{d_{1}}}\left(\mathcal{D}_{X}(A)\right) \times\left[-\pi, \pi\left[\times \mathbb{R}^{+} ; C_{A, B}^{\text {dist }}\left[d_{1}, d_{2}\right]\right.\right.\right.\right.\right.}\right\rangle
$$

The propagators associated with distance and direction constraints can be applied independently or combined to reduce the computational cost. For instance the propagator associated with the combination of constraints " $R R_{1}$ is on the right of $A_{4}$ and their distance is almost equal to the radius of $A_{4}$ " is illustrated in figure 5 . The domains of $A_{4}$ and $R R_{1}$ at a given step of the propagation process are respectively presented in (a) and (b). The propagator computes the set of points that satisfy the constraint with at least one element of $\mathcal{D}\left(A_{4}\right)(\mathrm{c})$ and updates the domain of $R R_{1}(\mathrm{~d})$ removing all values not in (c).

\subsubsection{Geodesic distance constraint}

Finally the model contains geodesic distance relations between control points that belong to the same vessels. Their distance along the vessel has to be smaller than a given value $\alpha$ (related to the prior distance between their position). As the vessels are not segmented, we consider an approximation of the geodesic distance relying on the results of the low-level filters (cf Section 3). We first compute a normalized ridgeness map $c$ whose values range between 0 and 1, 1 representing the membership to the vessel. The geodesic distance is then computed relatively to $\frac{1}{c(s)+\epsilon}$. 


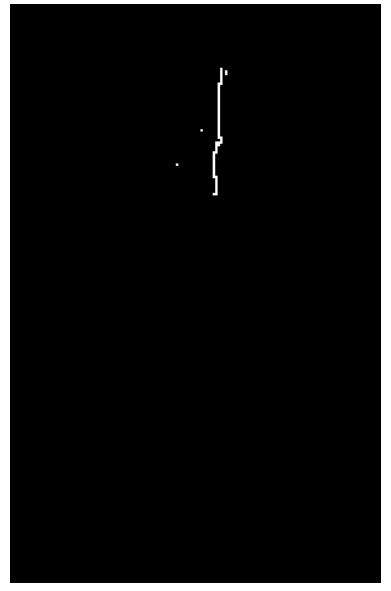

(a)

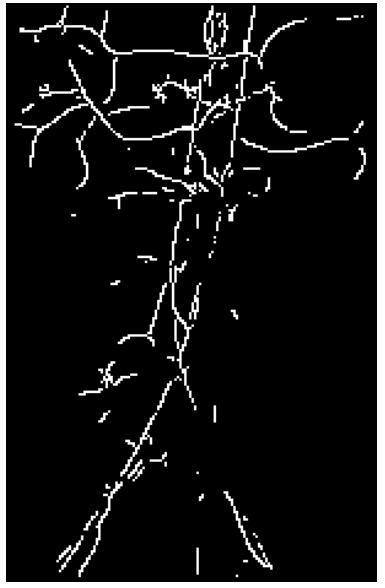

(b)

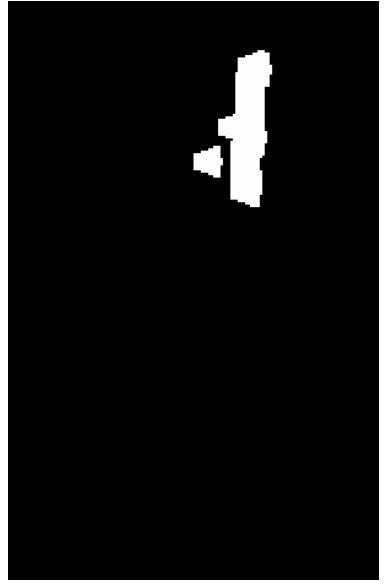

(c)

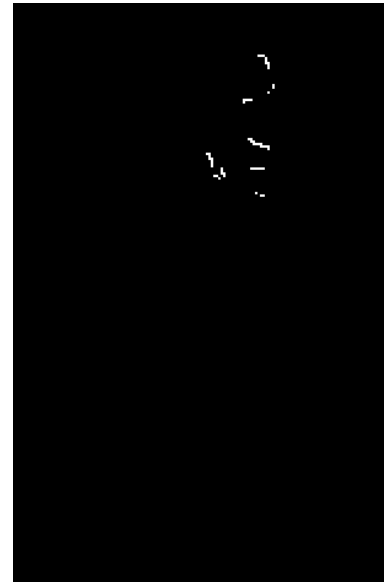

(d)

Figure 5. (a) $\mathcal{D}\left(A_{4}\right)$. (b) $\mathcal{D}\left(R R_{1}\right)$. (c) Values that satisfy the direction and distance constraints with at least one element of $\mathcal{D}\left(A_{4}\right)$. (d) Updated domain of $R R_{1}$.

Definition 4.5 (GeOdesic Distance COnstraint). Let $A$ and $B$ be two control points whose geodesic distance with respect to the vessel has to be smaller than $\alpha$. The constraint $C_{A, B}^{\text {path } \alpha}$ is defined as follows:

$$
\begin{aligned}
& \operatorname{rel}\left(C_{A, B}^{\text {path } \alpha}\right): \quad \mathcal{D}(A) \times \mathcal{D}(B) \quad \rightarrow\{0,1\} \\
& \left(\left(x_{1}, \theta_{1}, \sigma_{1}\right),\left(x_{2}, \theta_{2}, \sigma_{2}\right)\right) \mapsto \begin{cases}1 & \text { if } \inf _{l \in L_{x_{1}, x_{2}}} \int_{l} \frac{1}{c(s)+\epsilon} d s \leq \alpha \\
0 & \text { otherwise, }\end{cases}
\end{aligned}
$$

where $L_{x_{1}, x_{2}}$ denotes the set of paths from $x_{1}$ to $x_{2}$.

We denote by $d_{g}(x, y)$ the geodesic distance between $x$ and $y$ and by $d_{g}(x, S)$ the geodesic distance between $x$ and the set $S$. A value of $\mathcal{D}(B)$ satisfies the constraint with at least one value of $\mathcal{D}(A)$ if $\exists y \in \mathcal{D}_{X}(A), d_{g}(x, y) \leq$ $\alpha \Leftrightarrow d_{g}\left(x, \mathcal{D}_{X}(A)\right) \leq \alpha$. As $d_{g}\left(x, \mathcal{D}_{X}(A)\right)$ can be efficiently computed we obtain the following propagator.

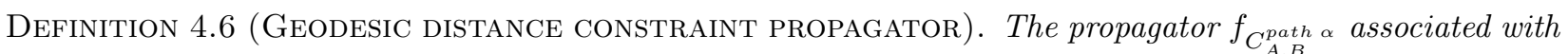
the geodesic distance constraint between two control points $A$ and $B$ is defined as follows:

$$
\frac{\left\langle A, B ; \mathcal{D}(A), \mathcal{D}(B) ; C_{A, B}^{\text {path } \alpha}\right\rangle}{\left\langle A, B ; \mathcal{D}(A) \cap\left\{(x, \theta, \sigma) \mid d_{g}\left(x, \mathcal{D}_{X}(B)\right) \leq \alpha\right\}, \mathcal{D}(B) \cap\left\{(x, \theta, \sigma) \mid d_{g}\left(x, \mathcal{D}_{X}(A)\right) \leq \alpha\right\} ; C_{A, B}^{\text {path } \alpha}\right\rangle}
$$

We illustrate this propagator in Figure 6. We consider a geodesic distance constraint $C_{A_{4}, A_{5}}^{\text {path }}$ between the control points $A_{4}$ and $A_{5}$ belonging to the aorta. The initial domains of those control points are respectively presented in (a) and (b). First we obtain the normalized ridgeness map $c(\mathrm{~d})$ to compute the geodesic distance from $\mathcal{D}_{X}\left(A_{4}\right): d_{g}\left(\mathcal{D}_{X}\left(A_{4}\right)\right)$. Then we remove from $\mathcal{D}\left(A_{5}\right)$ the values that do not satisfy $d_{g}\left(\mathcal{D}_{X}\left(A_{4}\right)\right) \leq \alpha$. We thus obtain the updated domain $\mathcal{D}_{X}{ }^{\prime}\left(A_{5}\right)(\mathrm{c})$.

\subsection{Propagation process}

The initial constraint network is build from the structural model and the domains are initialized by the results of the low-level filters. Moreover we assume that $A_{1}$ is close to the upper border of the X-Ray image and that $I L_{4}$ and $I R_{4}$ are close to the lower border. The propagators associated with the constraints are then iteratively applied by an $A C-3^{20}$ like algorithm presented in Figure 7 . It sequentially applies the propagators until convergence. As the propagators do not necessarily commute, they are generally applied more than once. Note also that the final result does not depend on the ordering of the propagators since they are monotonous.

This process is illustrated in Figure 8. We present the domains associated with the control points $R L_{1}, R R_{1}$ and $A_{4}$ (representing respectively the branching points of the left and right renal arteries and the point of the 


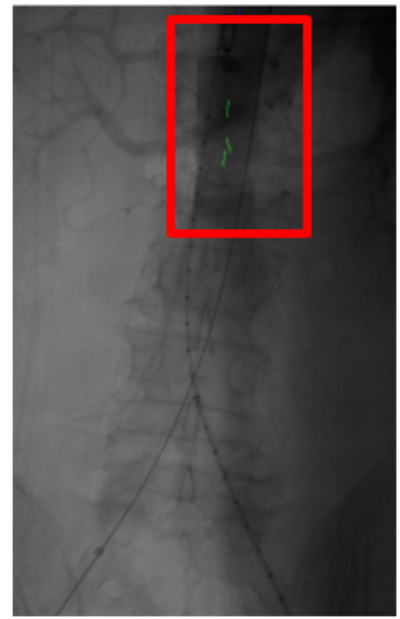

(a)

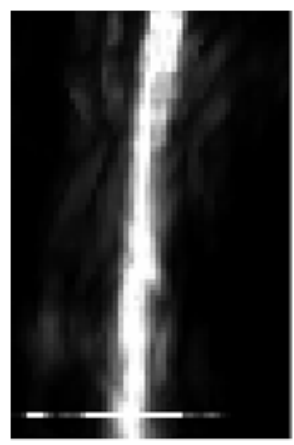

(d)

(e)

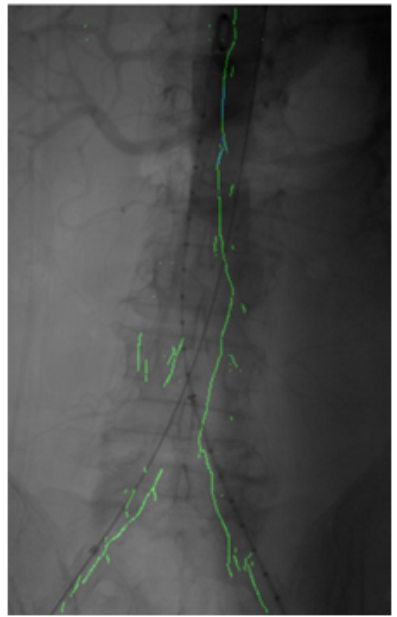

(b)

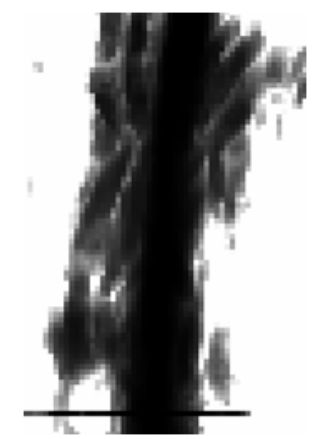

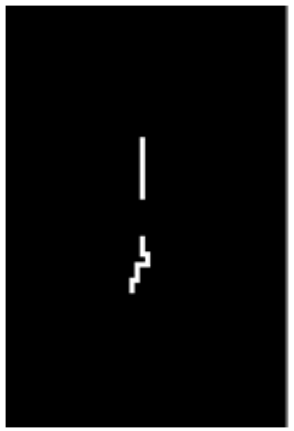

(f)

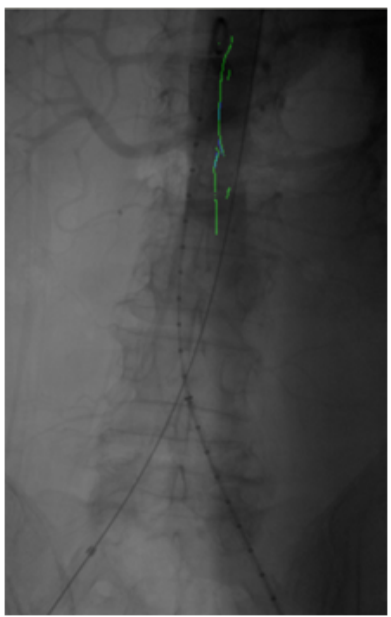

(c)

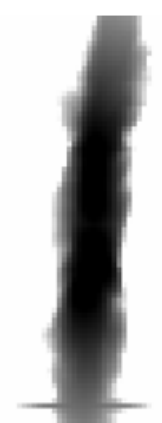

(g)

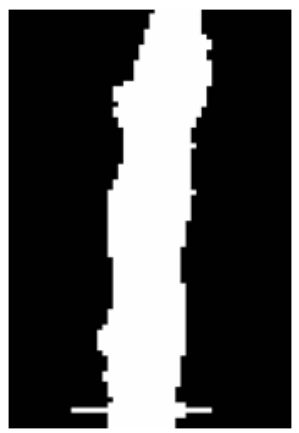

(h)

Figure 6. Illustration of the geodesic distance constraint between the control points $A_{4}$ and $A_{5}$ belonging to the aorta. Initial domains $\mathcal{D}_{X}\left(A_{4}\right)$ (a) and $\mathcal{D}_{X}\left(A_{5}\right)$ (b). Domain of $A_{5}$ updated by $f_{C_{A_{4}, A_{5}}^{\text {path }}}: \mathcal{D}_{X}{ }^{\prime}\left(A_{5}\right)$ (c). (d-h) Zoom on the red rectangle in (a). (d) Normalized ridgeness map c. (e) $\frac{1}{c(s)+\epsilon}$. (f) $\mathcal{D}_{X}\left(A_{4}\right)$. (g) $d_{g}\left(x, \mathcal{D}_{X}(A)\right)$. (h) $d_{g}\left(x, \mathcal{D}_{X}(A)\right) \leq \alpha$.

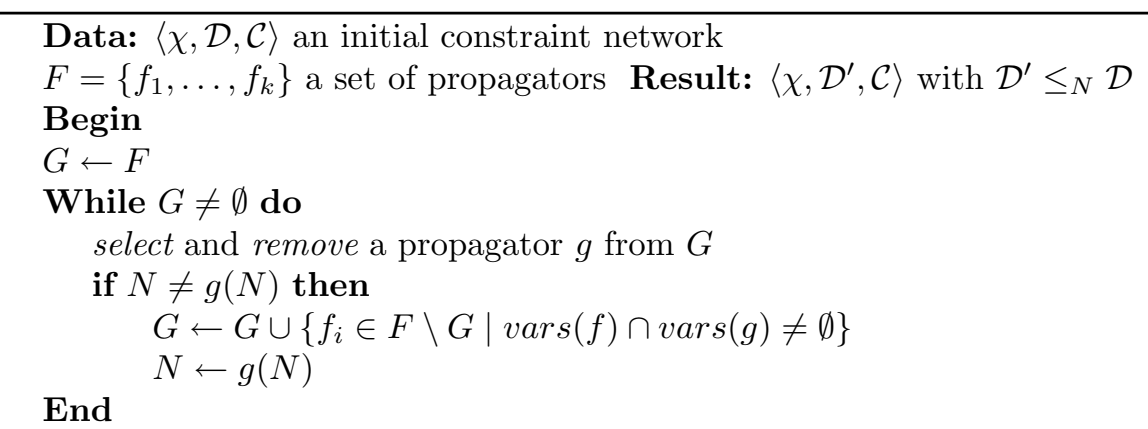

Figure 7. Generic $A C-3$ like constraint propagation algorithm.

aorta in front of the left renal artery) at several steps of the propagation process. Initially many possible positions are extracted by the low-level filters. The candidates for $A_{4}$ are presented in blue, whereas the possible positions for $R L_{1}$ and $R R_{1}$ are presented in yellow. The constraint propagation then progressively tightens the domains. We can observe that first the distance and directional constraints between $A_{4}$ and $R L_{1}$ allow to strongly reduce the domain of $R L_{1}$. Then further reductions of $\mathcal{D}\left(R L_{1}\right)$ allow to conversely obtain a reduction of $\mathcal{D}\left(A_{4}\right)$ using those constraints. At the end of the propagation process, only few candidates remain for those control points. 


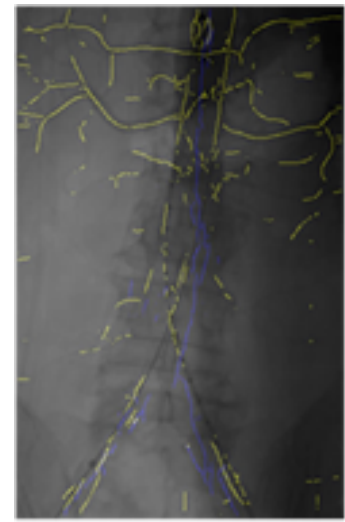

Iteration 0
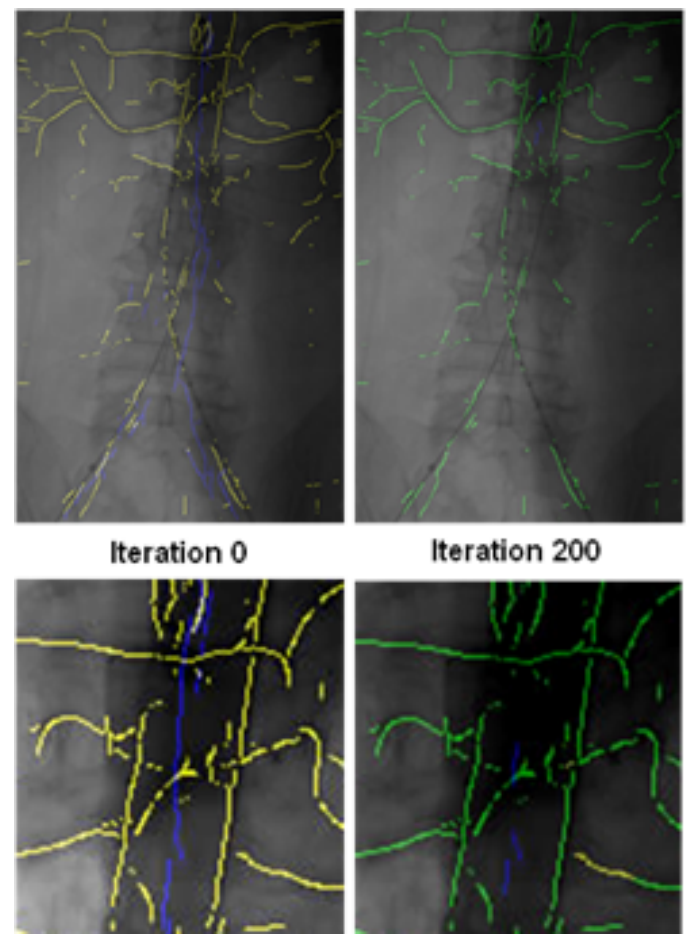

Iteration $\mathbf{2 0 0}$
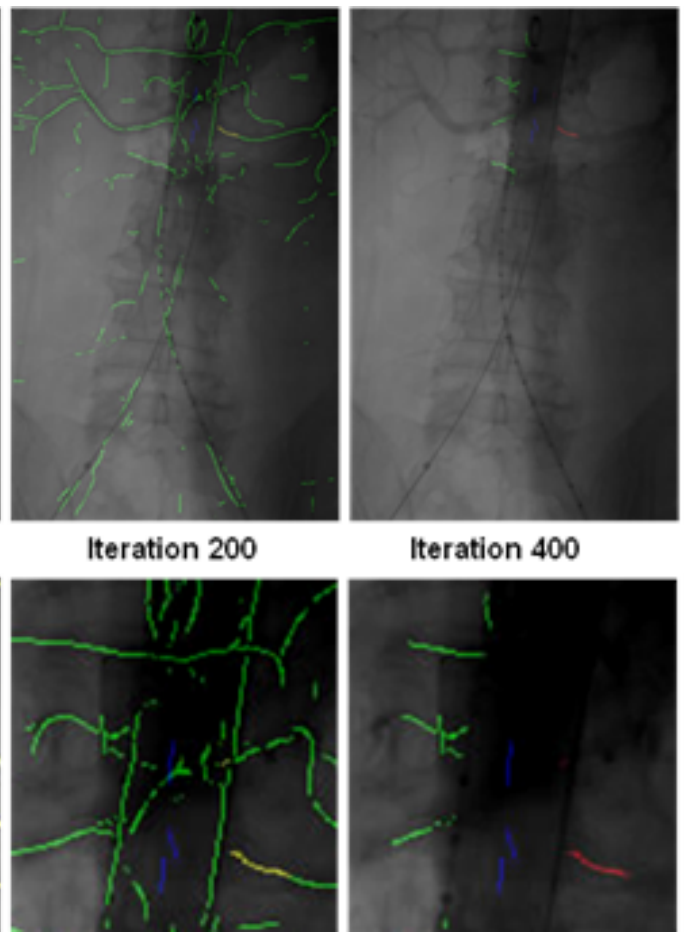

Iteration $\mathbf{4 0 0}$
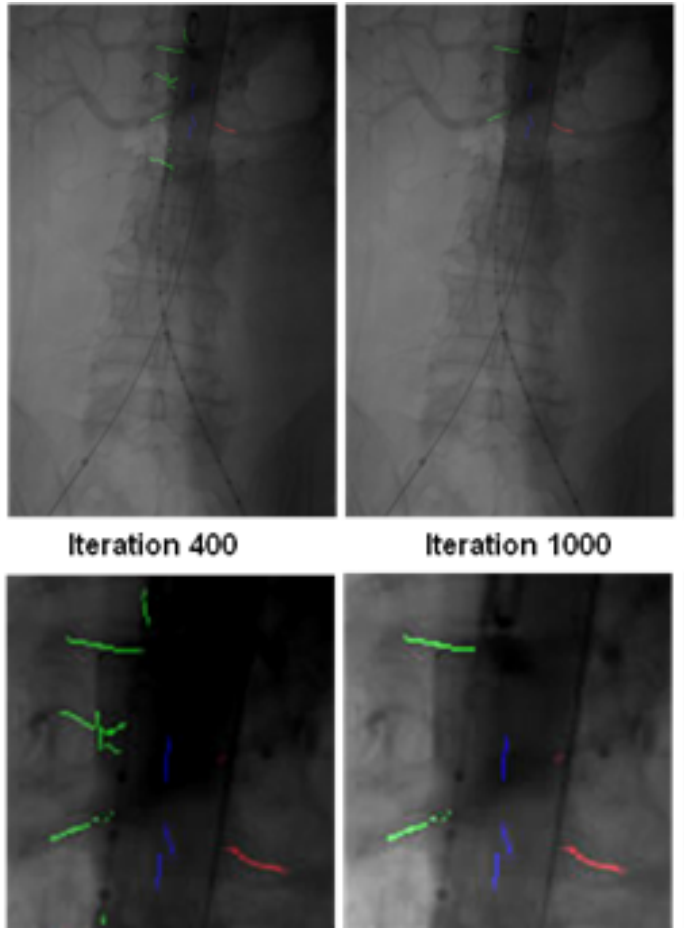

Iteration 1000
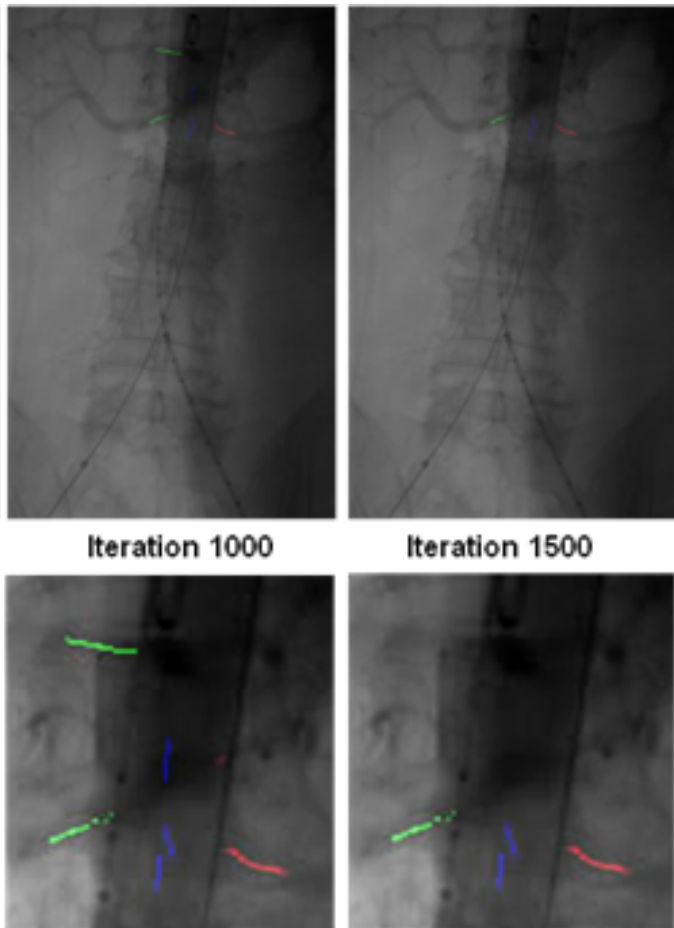

Iteration 1500

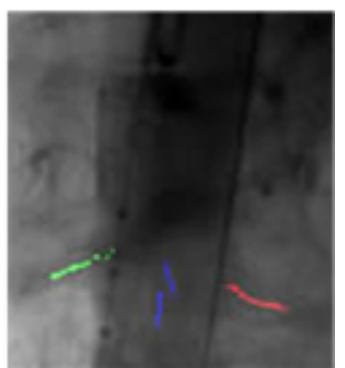

Figure 8. Domains associated with $R L_{1}$ (green), $R R_{1}$ (red) and $A_{4}$ (blue) at some steps of the propagation process.

\section{RESULTS}

We have manually build a model to perform the interpretation of angiograms centered on the abdominal aorta and including the renal arteries and the common iliac arteries. It contains 15 control points to represent the aorta, 15 to represent each renal artery, 10 to represent each common iliac and about 250 relations between those control points. Using this model, we evaluate the proposed approach on angiograms of 5 subjects acquired on Philips AlluraXper cardiovascular X-ray systems.

The process did not lead to inconsistency in any case and very few candidates remain in the resulting domains of the control points of interest. However, in some cases, ambiguities remain at the end of the process. For instance the domains of control points representing the left renal artery may contain values on left renal artery and on the splenic artery. The remaining possible instantiations should then be qualified to make a final decision. The whole process runs in about 2 minutes on a $512 \times 512$ image with a Pentium D 3.20GHz. Most of the computation time is spent in the constraint propagation algorithm, which may be optimized.

\section{CONCLUSION}

The use of a structural model allows to deal with the high variability of abdominal arteries. The structural model and the proposed recognition process based on low-level filtering and on constraint propagation seem to be well suited to achieve our recognition task. This approach is not prone to local minima, but several candidates generally remain at the end of the process and the computational cost is rather high. Future works aim at reducing the computational cost, setting up a final decision process, using fuzzy constraint network to make the model more specific on usual configurations and considering concurrently several models to perform the recognition task.

\section{REFERENCES}

[1] De Bruijne, M., Van Ginneken, B., Viergever, M., and Niessen, W., "Interactive segmentation of abdominal aortic aneurysms in CTA images," Medical Image Analysis 8(2), 127-138 (2004). 
[2] Olabarriaga, S., Rouet, J., Fradkin, M., Breeuwer, M., and Niessen, W., "Segmentation of Thrombus in Abdominal Aortic Aneurysms From CTA With Nonparametric Statistical Grey Level Appearance Modeling," IEEE transactions on medical imaging 24(4), 477-485 (2005).

[3] Bodur, O., Grady, L., Stillman, A., Setser, R., Funka-Lea, G., and ODonnell, T., "Semi-automatic aortic aneurysm analysis," in [SPIE], 6511, 65111G (feb 2007).

[4] Broit, C., Optimal registration of deformed images, $\mathrm{PhD}$ thesis, University of Pennsylvania, Philadelphia (aug 1981).

[5] Cootes, T., Edwards, G., and Taylor, C., "Active Appearance Models," IEEE Transactions on Pattern Analysis and Machine Intelligence 23, 681-685 (jun 2001).

[6] De Bruijne, M., Van Ginneken, B., Viergever, M., and Niessen, W., "Adapting Active Shape Models for 3D Segmentation of Tubular Structures in Medical Images," in [Information Processing in Medical Imaging], LNCS 2732, 136-147, Springer, Ambleside, UK (jul 2003).

[7] Bloch, I., Géraud, T., and Maître, H., "Representation and Fusion of Heterogeneous Fuzzy Information in the 3D Space for Model-Based Structural Recognition - Application to 3D Brain Imaging," Artificial Intelligence 148, 141-175 (2003)

[8] Bessière, C., "Constraint Propagation," tech. rep., LIRMM, UMR 5506 CNRS, University of Montpellier (2006).

[9] Deruyver, A. and Hodé, Y., "Constraint satisfaction problem with bilevel constraint: application to interpretation of over-segmented images," Artificial Intelligence 93(1-2), 321-335 (1997).

[10] Nempont, O., Atif, J., Angelini, E., and Bloch, I., "Structure Segmentation and Recognition in Images Guided by Structural Constraint Propagation," in [European Conference on Artificial Intelligence ECAI], 621-625 (jul 2008).

[11] Hodé, Y. and Deruyver, A., "Qualitative spatial relationships for image interpretation by using semantic graph," in [Graph-Based Representations in Pattern Recognition, GbRPR], 240-250 (2007).

[12] Eberly, D., Gardner, R., Morse, B., Pizer, S., and Scharlach, C., "Ridges for Image Analysis," Journal of Mathematical Imaging and Vision 4(4), 353-373 (1994).

[13] Frangi, A. F., Frangi, R. F., Niessen, W. J., Vincken, K. L., and Viergever, M. A., "Multiscale vessel enhancement filtering," in [Medical Image Computing and Computer-Assisted Interventation, MICCAI], LNCS 1496, 130-137, Springer-Verlag, Cambridge, MA, USA (oct 1998).

[14] Jacob, M., Unser, M., et al., "Design of steerable filters for feature detection using canny-like criteria," IEEE transactions on pattern analysis and machine intelligence 26(8), 1007-1019 (2004).

[15] Apt, K., [Principles of Constraint Programming], Cambridge Univ Pr, New York, NY, USA (2003).

[16] Rossi, F., Van Beek, P., and Walsh, T., eds., [Handbook of Constraint Programming], Elsevier, New York, NY, USA (2006).

[17] Golomb, S. and Baumert, L., "Backtrack Programming," Journal of the ACM, JACM 12, 516-524 (oct 1965).

[18] Rossi, F., Van Beek, P., and Walsh, T., [Handbook of Constraint Programming], ch. 4, Backtracking search algorithms, Elsevier (2006).

[19] Bloch, I., "Fuzzy Relative Position between Objects in Image Processing: a Morphological Approach," IEEE Transactions on Pattern Analysis and Machine Intelligence 21, 657-664 (jul 1999).

[20] Mackworth, A., "Consistency in networks of relations," Artificial Intelligence 8, 99-118 (feb 1977). 